\title{
Anisoplanatism effects in Shack-Hartmann Wave Front Sensing with Laser Guide Stars on the ELTs
}

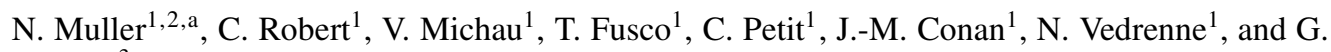 \\ Rousset $^{3}$ \\ 1 Onera, DOTA / HRA, Chatillon, France \\ 2 Groupement d'Intéret Scientifique PHASE (Partenariat Haute résolution Angulaire Sol Espace) be- \\ tween Onera, Observatoire de Paris, CNRS and Université Paris Diderot \\ 3 Observatoire de Paris-Meudon, LESIA, Meudon, France
}

\begin{abstract}
Laser Guide Stars aim at increasing the sky coverage, highly restricted when using Adaptive Optics with Natural Guide Stars. With such an artificial object, spot elongation may be a limitation for the measurement quality when using a Wave Front Sensor. In this document, we evaluate and discuss the effects of this limitation on wave front measurements, for a $40 \mathrm{~m}$ class telescope. We also explore the possibilities of taking these effects into account in the wave front reconstruction.
\end{abstract}

\section{Introduction}

Adaptive Optics (AO) relies on a Wave Front Sensor (WFS) to measure properly the pertubations induced by the turbulence on the wavefront. Yet, source extension may limit its performance: waves coming from different points of an extended source, such as Laser Guide Stars (LGS), are not identically distorted by the turbulence, depending on the atmospheric volume seen through the propagation. This effect, called phase anisoplanatism, refers to an angular decorrelation of the phase perturbations in the Field of View (FoV) ([1], [2]). Next to phase anisoplanatism, scintillation anisoplanatism accounts for the angular dependence of the collected flux in the pupil plane. These effects both affect the accuracy of the measurements. On an extended source, the measurement accuracy for astronomical, solar and endo-atmospheric observations [3], and its influence on the wavefront estimation [4] have been investigated as well.

Measurement accuracy on an extended object is an issue when AO systems, such as Multi Conjugated (MCAO) systems, are used with LGS [5], especially for the next generation of $40 \mathrm{~m}$ telescopes, called Extremely Large Telescopes (ELTs). Indeed, by focusing a CW laser beam in the atmospheric sodium layer, we obtain a pin-like laser beacon, around $10 \mathrm{~km}$ long, which is seen from the edge of the pupil with a field angle that increases with the diameter of the telescope (typically 5 to 10 arcsec with an ELT).

The present study is aimed at estimating the wave front measurement error induced by phase and scintillation anisoplanatism, in case of LGS for the ELTs. We select the Shack-Hartmann WFS (SH WFS), which is the most commonly used nowadays. We propose to evaluate the impact of the anisoplanatic error first on the measurement precision at the subaperture level, then on the reconstructed wavefront, when using a SH WFS on one LGS or more.

Our approach is to evaluate firstly the measurement error with a numerical model, to check the results with an analytical model, and then to use the measurement error thus estimated in the wavefront reconstruction process. For this purpose, we start by presenting the numerical and analytical models in Sect. 2, showing both results in comparison. Then we present in Sect. 3 the formalism used for the reconstruction, as well as numerical estimations of the wavefront reconstruction error for both single and multi LGS cases.

\footnotetext{
a e-mail: nicolas.muller@onera.fr
} the original work is properly cited. 


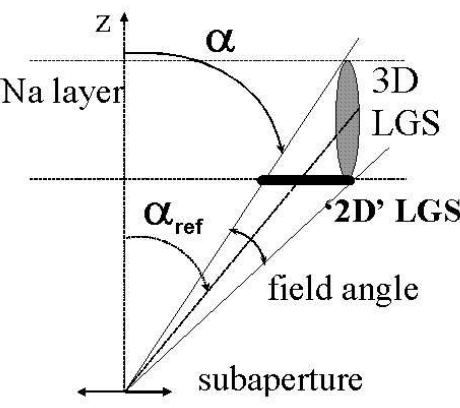

(a)

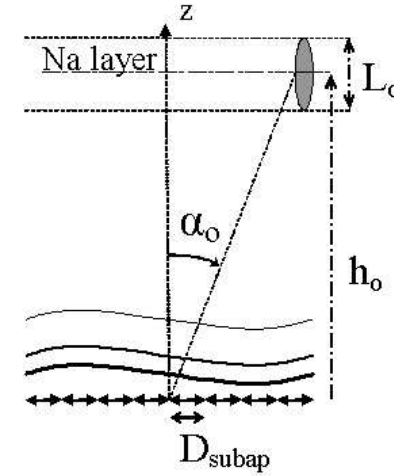

(b)

Fig. 1. Main parameters of the study. (a) Principle of the 2D analytical model. (b) Principle of the 3D numerical model : example of a 1:2 downscaled model. The $C_{n}^{2}$ profile is unchanged from the 1:1 to the 1:2 model.

\section{Anisoplanatic slope measurement error}

In this study we consider a SH WFS with a fixed subaperture size of 0.5 meters, as set by the first studies of ELT systems [6] [7] [8]. Since we are only interested in the evaluation of the anisoplanatism impact, we consider a noise-free case. The slope estimator is the basic center of gravity $(\mathrm{CoG})$ and is always computed on Shannon-sampled images. The measurement error is denoted by the fluctuations of the global $\mathrm{CoG}$ of the extended object, referenced to the fluctuations of the CoG that would be calculated for a point source in the particular direction of the center of the object. A complete description of the anisoplanatic slope measurement error can be found in [3]. Other algorithms will be tested in future work.

\subsection{Parameters of the simulations}

In order to simulate the diffractive propagation, we use the PILOT code, developed at Onera. Coupled to a SH WFS measurement simulator, it allows us to estimate here the slope measurement error made when using a SH WFS on a LGS. Further details on this algorithm can be found in [3] and [4]. In order to validate the numerical results obtained with this $3 \mathrm{D}$ end-to-end simulation, we use a $2 \mathrm{D}$ analytical model. This model allows us to study the measurement error that would be induced by an horizontal object located at the lowest altitude of the sodium layer (see fig. 1(a)), with the same field angle as the original one. It is described more in details in [3]. Both models, numerical and analytical, are able to take into account phase anisoplanatism effects only, scintillation effects only, or both effects together.

$3 \mathrm{D}$ simulations on a $42 \mathrm{~m}$ telescope being too heavy to be run with the means currently at our disposal, we choose to run them on downscaled cases, where all the distances are reduced by a factor 2 (1:2 model) or 3 (1:3 model), but with all the angles conserved, as well as the size of the subaperture, and the $C_{n}^{2}$ profile, in terms of altitude and strength (see fig. 1(b)). In this configuration geometrical effects are unchanged: the turbulence profile remains the same so that scintillation is still modelized correctly. The agreement between the downscaled results and the original ones has been checked. The 3D simulation is run on a 1:2 model, while the analytical model is run on a 1:1 model. The Na layer is set uniform, $10 \mathrm{~km}$ thick, leading to a maximum elongation of $\approx 10 \mathrm{arcsec}$. We have evaluated the convergence of the results as the sampling of the LGS elongation increases. This lead us to choose a LGS sampling of 9 points for our simulations. A similar estimation for the sampling of the volume of turbulence makes us choose either the 9-layer ESO profiles [9] or an equivalent 5-layer profile for 


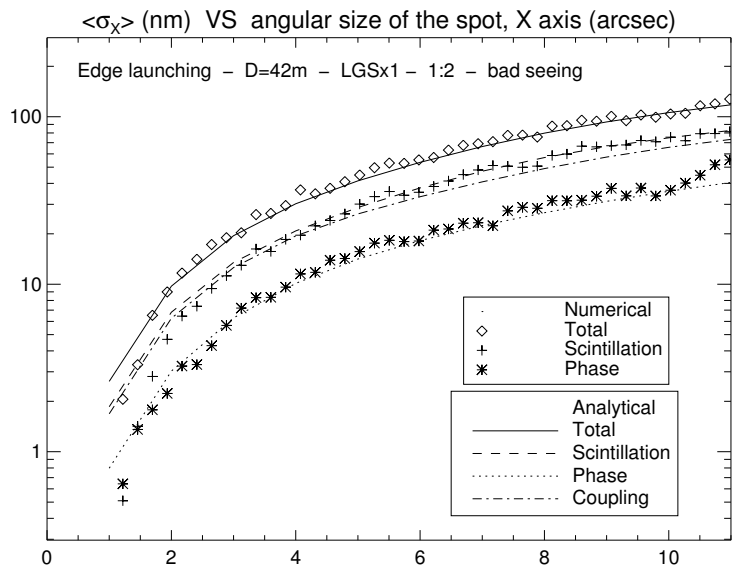

Fig. 2. Measurement error standard deviation along the $X$ axis $(\mathrm{nm})$ in function of the angular size of the spot along the $\mathrm{X}$ axis $(\operatorname{arcsec})$ ) contribution of the different terms. The errors obtained analytically with phase (scintillation) effects only are represented by a dotted (dashed) curve. The total error of both effects plus the coupling (dash-dotted curve) is represented for comparison (solid curve). The errors obtained numerically with phase (scintillation) effects only are denoted by stars (crosses). Those obtained with both effects are denoted by diamonds.

our simulations. We use preferentially the profiles corresponding to a good seeing (0.6 arcsec) or bad seeing (1.1 arcsec), as they set the lower and higher limits of the error with regards to the turbulence strength. Finally, around a hundred propagations were performed in our Monte-Carlo simulations.

\subsection{Results}

Results shown in Fig. 2 are obtained for a single LGS, launched on the X axis, from the edge of the primary mirror of a 42-meter telescope. They show the measurement error in the sub-pupils along the $\mathrm{X}$ axis, depending on the spot elongation on $\mathrm{X}$.

The results show a good agreement between the 2D and 3D approaches, which validates our downscaled model. Then, analytical and numerical results both show the preponderance of scintillation anisoplanatism and coupling in the total error, while the phase anisoplanatism is almost negligible, no matter how elongated the spot may be. The measurement error, obtained with bad seeing conditions, reaches $\approx 100 \mathrm{~nm}$ in standard deviation $\left(\approx 1 \mathrm{rad}^{2}\right.$ in variance $)$ in the subapertures where the spot elongation is maximum, i.e. the subapertures diametrically opposed to the laser launching point. Taking into account a good seeing, the level of the error roughly decreases by a factor 2 in variance. Nevertheless, this error has to be included into a reconstruction process, in order to estimate the effective impact of anisoplanatism on the system.

\section{Wave front reconstruction error}

In this section, we focus on the wave front reconstruction, and on the phase error in the telescope pupil, further called Wave Front Error (WFE). We aim at computing the propagation of the anisoplanatic slope measurement error (at subaperture level) through the reconstruction, and evaluating the WFE in a single LGS case, and a 6 LGS case. We will test LS (Least Squares), WLS (Weighted LS), MAP (Maximum A Posteriori) and Unif.MAP (MAP with Uniform Noise) reconstruction.

\subsection{Principle of the calculations}

Let's consider the slope error covariance matrix $C_{\epsilon}$, concatenating the slope measurement error variances of each subaperture, the error covariances for each couple of subapertures, and the correlations 
between each LGS in a multi-LGS scheme. The SH WFS being assumed as linear, the measured slopes $S$ may be expressed as a function of the phase $\Phi$ coming from one LGS, so that $S=D \Phi+\epsilon$. $D$ is the interaction matrix giving the SH response to the phase and $\epsilon$ the slope error. In the following, we choose to expand the phase on the KL basis so that $\Phi$ is the KL coefficient vector.

Considering a WLS reconstructor, the estimated phase is given by $\hat{\Phi}=R S$, with $R$ the reconstruction matrix [10], written as $R=\left(D^{T} C_{\epsilon}^{-1} D\right)^{-1} \cdot D^{T} C_{\epsilon}^{-1}$. The phase error in the telescope pupil is then given by the sum of the KL noise variances, i.e. the diagonal terms of the error covariance matrix of the phase:

$$
W F E=\sigma_{\epsilon}^{2}=\sum_{i} \operatorname{Var}\left(\hat{\Phi}_{i}\right)=\operatorname{trace}\left(R C_{\epsilon} R^{T}\right)
$$

In case of a multi-LGS launching scheme, a tomographic reconstruction of the volume of turbulence is required [11]. For sake of simplicity, the slope error is here assumed to be independant of the phase, as a preliminary approach. The interaction matrix $D_{\alpha}^{\prime}=D^{\prime} M_{\alpha}$ is first calculated by multiplying $D^{\prime}$, derived from the $\mathrm{D}$ matrix, with $M_{\alpha}$, a projection matrix which accounts for the different directions $\alpha_{i}$ of the LGS in the FoV, i.e. the directions where the turbulent volume is probed. In case of a MAP reconstructor, the tomographic reconstruction matrix $W$ is then given by:

$$
W=\left(D_{\alpha}^{\prime T} C_{\epsilon}^{-1} D_{\alpha}^{\prime}+C_{\phi}^{-1}\right)^{-1} \cdot D_{\alpha}^{\prime T} C_{\epsilon}^{-1}
$$

where $C_{\phi}$ is the turbulence covariance matrix, giving the a priori on the turbulent volume. Yet the MAP reconstructor is hard to use here in practice, since it assumes that the $C_{n}^{2}$ profile and the error are known precisely. Using Unif.MAP (or LS instead of WLS in the previous case) sets the error covariance matrix to $\sigma^{2} \times \mathbb{I}$, where $\mathbb{I}$ is the identity matrix and $\sigma^{2}$ a reference error set to the measurement error variance obtained with maximum elongation. The reconstructed phase in any of the directions $\beta_{0}$ of the FoV, $\Phi_{\beta_{0}}$, is obtained by projection in this particular direction, so that $\Phi_{\beta_{0}}=M_{\beta_{0}} W S$. Finally, the phase error in this direction is given by:

$$
W F E=\operatorname{trace}\left(M_{\beta_{0}} W C_{\epsilon} W^{T} M_{\beta_{0}}^{T}\right)+\operatorname{trace}\left(M_{\beta_{0}} B M_{\beta_{0}}^{T}\right)
$$

where $B=\left(W D_{\alpha}^{\prime}-\mathbb{I}\right) C_{\phi}\left(W D_{\alpha}^{\prime}-\mathbb{I}\right)^{T}$ is the bias of the reconstructor. For this study our approach is to calculate the covariance matrix $C_{\epsilon}$ with a MC method based on the end-to-end simulation, and then to evaluate the WFE with the formulas (1) or (3). However, due to the coupling between the error and the phase, the reconstruction is not optimised, and the WFE slightly inappropriate.

\subsection{Single-LGS reconstruction}

We choose a model where the LGS is launched vertically, from the edge of M1, at an angle of 48 arcsec from the center of the FoV, in a MAORY-like configuration for a single LGS [6]. We use a 1:2 model and perform the reconstruction of the phase in the pupil, on the 1000 first K-L modes, considering a telescope without central occultation. The LGS is sampled with 9 point sources. The $C_{n}^{2}$ profile corresponds to bad seeing conditions, and is reduced to an equivalent 5-layer profile in that case in order to speed up calculations. Since the simulations are very time and memory consuming, the error covariance matrix $C_{\epsilon}$ is still quite noisy, with values having a very low SNR, which makes its inversion difficult. Consequently we set to zero the elements of the matrix that are above a given threshold. Fig. 3 shows the standard deviation of the reconstructed phase error for various reconstructors.

Let's first notice that results obtained with WLS and MAP are very close one to each other. This means that the SNR is high, so that the influence of the prior on the phase (i.e. the $C_{\phi}$ matrix, see Eq. 2) is negligible. As it is the same for the LS and Unif.MAP reconstructors, we show only LS and WLS results here. The validity of the approximation of independance between error and phase is somewhat reinforced by these results.

Secondly, results obtained with WLS and MAP reconstructors are limited by the noise level in the error covariance matrix $C_{\epsilon}$. Yet we can see that using these reconstructors makes the error decrease, 


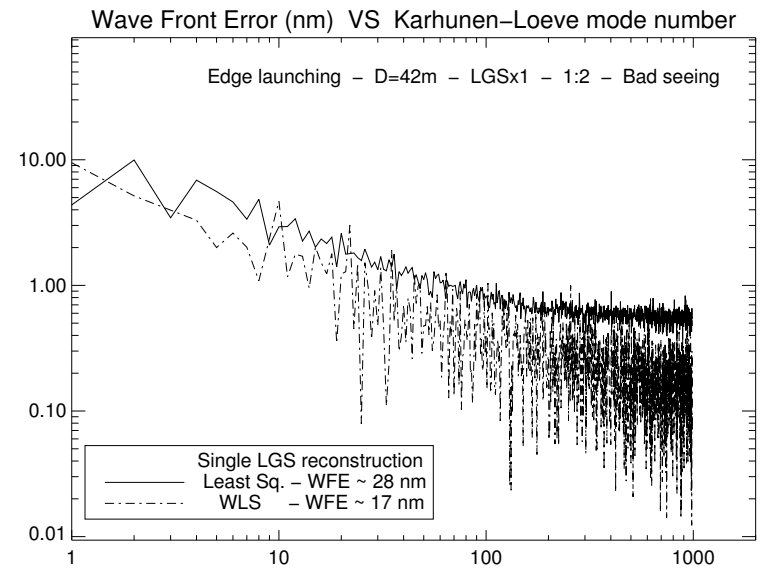

Fig. 3. Reconstruction error $(\mathrm{nm})$ in function of the Karhunen-Loeve mode number: LS and WLS reconstructors for a single side-launched LGS. The scale of the model is 1:2. The error on the reconstructed phase is shown in solid or dash-dotted curve depending on the reconstructor used, LS or WLS respectively.

and helps reduce the total wave front error from $\approx 30 \mathrm{~nm}$, when using a LS or Unif.MAP reconstructor in that case, to $\approx 20 \mathrm{~nm}$.

All in all, the error comes essentially from the slope measurement error along the elongated dimension, as seen from each subaperture, since the impact of scintillation is negligible in the perpendicular direction [3]. This way, using multiple LGS should reduce the impact of the error, as it is the case for the detector noise [12].

\subsection{Multi-LGS tomographic reconstruction}

The launching scheme for these simulations is the same as the one used for a single LGS case [6]. We choose a 6 LGS configuration, equally distributed, in a MAORY-like configuration. The reconstruction precision is estimated in the direction $\beta_{0}$ that corresponds to the center of the FoV. The scale of the model is now reduced to 1:3 so that the simulations are technically feasible with 6 LGS. We still use a thresholded covariance matrix in the standard case (cf. Subsect. 3.2). We also run the simulations in the particular case where we reduce the error covariance matrix $C_{\epsilon}$ to a diagonal matrix by setting all its non-diagonal elements to zero, in order to study the impact of the cross-correlation terms on the estimation of the error. Fig. 4 shows the error standard deviation of the reconstructed phase on the KL modes, in a tomographic reconstruction process using a MAP reconstructor, for these various cases.

Results obtained with a diagonal error covariance matrix roughly show a gain of a factor 2 to 3 when passing from a single LGS case to a multi LGS case, which seems realistic when crosschecking with results of other studies [12] [13]. Orders of magnitude of the WFE over 300 modes in that case are around 20 to $30 \mathrm{~nm}$ for a single LGS, and around $10 \mathrm{~nm}$ for a multi LGS case.

Results obtained with the thresholded covariance matrix are noisy. Yet we can see that the errors on the reconstructed phase for either 1 or 6 LGS, are very close to each other. Moreover they are closer than in the previous case, when $C_{\epsilon}$ was diagonal. This makes us think that the cross-correlation terms have a non-negligible impact on the error propagation. All in all, the error on the reconstructed phase using 6 LGS is somehow of the same order of magnitude than the single LGS case, around 10 to 20 $\mathrm{nm}$.

\section{Conclusion}

In this study, we evaluated the order of magnitude of the anisoplanatic slope measurement error that is made when using a SH WFS on a side-launched LGS, on a 42-meter telescope, and highlighted 


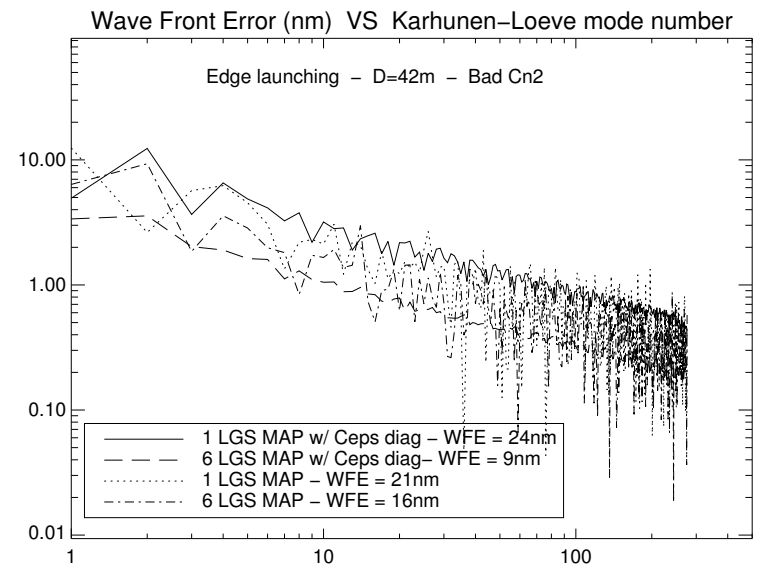

Fig. 4. Tomographic reconstruction error $(\mathrm{nm})$ in function of the Karhunen-Loeve mode number: MAP reconstructor for 1 or 6 side-launched LGS, with $C_{\epsilon}$ being diagonal or thresholded. The scale of the model is 1:3. The errors obtained for a single (multi) LGS case with a diagonal $C_{\epsilon}$ are shown in bold face solid (bold face dashed) curve, the ones obtained with a thresholded $C_{\epsilon}$ are shown in dotted (dash-dotted) curve.

the importance of the scintillation and coupling in these results. We also presented the propagation of this anisoplanatic error on the reconstructed phase, for a single side-launched LGS channel and for a multi-LGS case, using different reconstructors, with or without priors.

It appears that the anisoplanatic slope measurement error can go up to $100 \mathrm{~nm}\left(1 \mathrm{rad}^{2}\right)$ under bad seeing conditions, leading to a WFE of a few dozens of nanometers when using a LS reconstructor under the same conditions. Adding information about the noise covariance lowers these errors even more. A multi-LGS scheme gives the same orders of magnitude for the error on the reconstructed phase as a single LGS case. Yet these estimations remains uncertain since we face a lack of precision on our error covariance matrix, due to a rather small number of propagations in our Monte-Carlo simulations, and base our calculations on an inappropriate approximation concerning the independance between error and phase. Future work tends to tackle these problems.

\section{Acknowledgments}

This work has been performed in the framework of a Ph.D thesis supported by the French Délégation Générale pour l'Armement.

\section{References}

1. F. Chassat, J. Opt. (Paris) 20, 13-23 (1989)

2. R.J. Sasiela, Electromagnetic Wave Propagation in Turbulence, Springer-Verlag (1994)

3. C. Robert et al., J. Opt. Soc. Am. A 23, (2006) 613-624

4. N Vedrenne et al., J. Opt. Soc. Am. A 24, (2007) 2980-2993

5. F. Rigaut et al., C.R. Physique 6 (2005)

6. E. Diolaiti, Proc. of the AOforELT conference, (2009)

7. T. Fusco, Proc. of the AOforELT conference, (2009)

8. G. Rousset, Proc. of the AOforELT conference, (2009)

9. S. Oberti, E-SPE-ESO-276-0206 issue1 (2008)

10. B. L. Ellerbroek, J. Opt. Soc. Am. A 11, (1994) 783-805

11. T. Fusco et al., J. Opt. Soc. Am. A 18, (2001) 2527-2538

12. M. Tallon et al., SPIE 7015, 7015N (2008)

13. C. Robert, Proc. of the AOforELT conference, (2009) 